\title{
Combinaison d'une soupape de sûreté et d'une soupape de by-pass haute pression dans les installations de chaudières à circulation forcée
}

\author{
Combined safety valve and by-pass valve \\ in forced-feed boiler installations
}

\author{
M. Grotloh
}

Sulzer Frères, Winterthur

Avantages du fait de combiner les soupapes de sûreté et de by-pass

Une soupape de sûreté, dans le sens classique, est un dispositif de protection contre le dépassement de la pression de service maximale autorisée. Elle possède une régulation de pression mécanique sans réglage de la valeur de consigne et dans la mesure du possible, n'est jamais utilisée mais doit pouvoir "cracher" la totalité du débit de vapeur produit.

Mais, à côté de cela, un générateur de vapeur de centrale nécessite encore d'autres fonctions de protection. Ceci en particulier lorsqu'un délestage et une fermeture rapide de la turbine ne peuvent provoquer un arrêt automatique du foyer. Les exigences suivantes doivent être assurées :

- les surfaces de chauffe haute pression conduisant la vapeur ne doivent pas être surchauffées pendant le processus de démarrage et le service à charge partielle. Les soupapes de sûreté réglées sur une valeur de réponse fixe n'offrent donc aucune sécurité dans le cas d'une situation à pression glissante. Elles devraient également présenter une pression de réponse décroissante corres. pondante avec une pression dégressive de la chaudière; - le resurchauffeur doit être suffisamment refroidi, c'est-à-dire balayé. Le débit manquant de la turbine haute pression est amené au resurchauffeur par la vapeur déchargée côté haute pression;

- le resurchauffeur doit être protégé même dans le cas de basses pressions partielles. Le dispositif de by-pass basse pression doit donc également être équipé avec une pression de réponse glissante.

Donc une connexion doit être créée entre la conduite haute pression et le collecteur froid du resurchauffeur. Elle existe déjà généralement sous la forme d'un by-pass haute pression qui est utilisé pour le démarrage et l'arrêt. Contrairement aux chaudières à circulation forcée, il n'est souvent pas, sur les chaudières à ballon, conçu pour la capacité à pleine charge (Fig. 1). Ce by-pass est doté d'une régulation de pression de haute qualité. La valeur de consigne peut être réglée manuellement ou automatiquement. La température de la vapeur qui chute du fait du processus d'expansion est réduite par injection et mélange intensif d'eau à la température d'entrée du resurchauffeur.
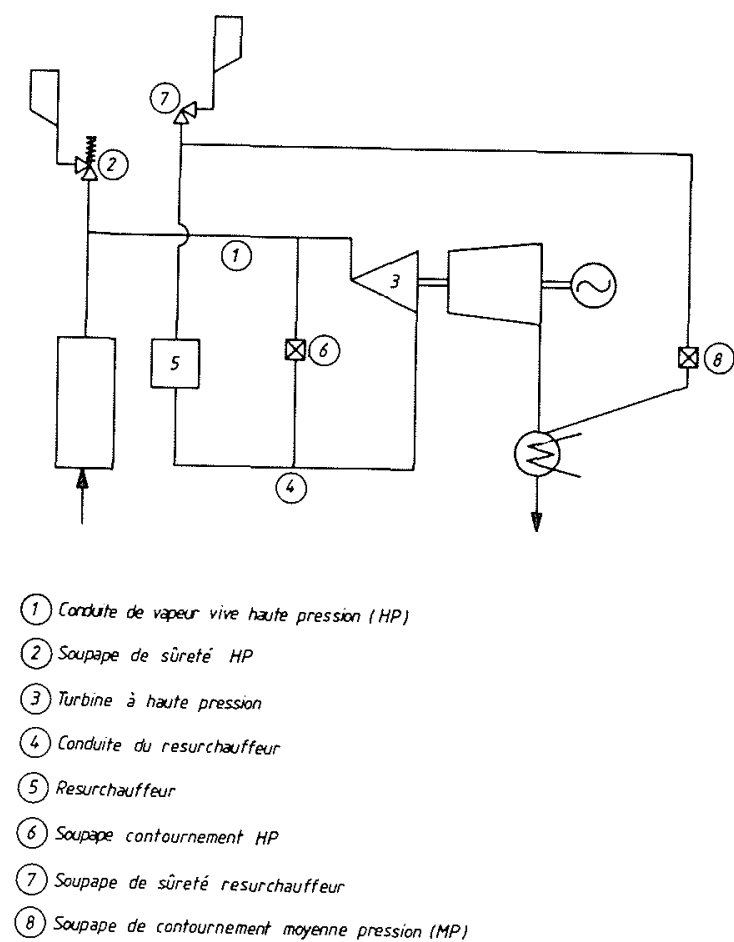

Figure 1

L'idée de combiner la soupape de sûreté et la soupage de by-pass haute pression va alors de soi (Fig. 2). Natu- 


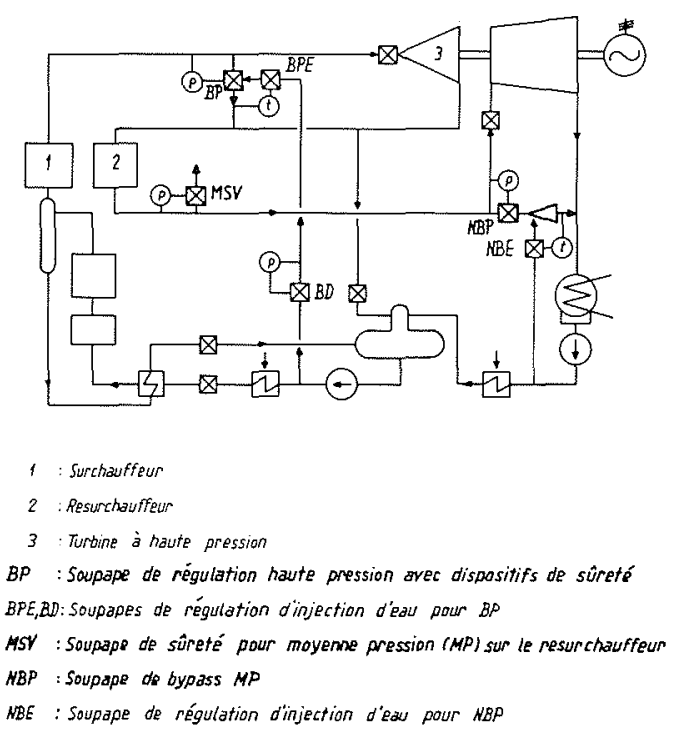

Figure 2. - Schéma de connexion de la soupape de contournement avec dispositifs de régulation et de sûreté pour chaudières à circulation forcée

rellement, aucune des exigences fondamentales, telles que - rapidité et sûreté d'ouverture, - rapidité et sureté d'amenée d'eau d'injection, - conception pour $100 \%$ du débit, ne doit être ignorée.

Les avantages sont les suivants :

- démarrage optimal et rapide de la chaudière et de la turbine ;

- passage à travers le resurchauffeur et en conséquence sa protection dans le cas d'une fermeture rapide de la turbine;

- la chaudière peut rester en service avec les vannes de la turbine fermées;

- pas de "crachement" des soupapes de sûreté dans l'atmosphère et en conséquence réduction des nuisances de bruit dans les régions à forte densité urbaine ;

- élimination des pertes d'eau entrant en considération dans le cas d'un crachement prolongé dans l'atmosphère.

Les-missions de la soupape de sûreté et de by-pass combinés sont les suivantes :

- prévention des dépassements de pression non autorisés dans la chaudière en toutes circonstances ;

- remplir la fonction des vannes de sûreté avec une pression de réponse décroissante dans le cas d'un délestage avec un mode de fonctionnement à pression glissante; - régulation de la pression de vapeur vive lors du démarrage et de l'arrêt ;

- adaptation de la température pendant le démarrage, l'arrêt, ainsi que pendant les perturbations de la charge par injection d'eau.

Description de l'exécution constructive d'une soupape de by-pass haute pression/sûre té combinée (Fig. 3)

La soupape est formée d'un corps forgé, sur lequel sont soudées les tubulures d'entrée et de sortie. Le siège de la soupape est disposé entre les deux sphères qui

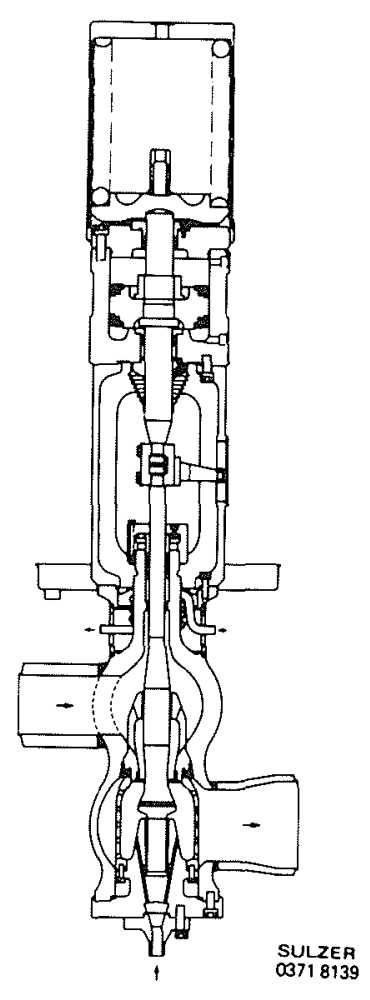

Figure 3. - Soupape de contournement haute pression

forment le corps. L'obturateur, situé du côté basse pression, sert de guide à la tige de soupape et porte les buses d'injection d'eau et la cage brise-jet de sortie. Cette soupape est commandée par un servomoteur hydraulique à double effet, comprenant en plus un ressort d'ouverture. Le déclenchement du dispositif de commande provoque l'ouverture d'un by-pass à l'huile entre les chambres supérieure et inférieure du piston. C'est ensuite l'effort fourni par la vapeur - secondé par celui du ressort - qui ouvre la soupape de by-pass haute pression.

Le constructeur a attaché la plus grande attention à la forme du corps de la soupape. Il doit non seulement résister aux sollicitations statiques de pression et température, mais également aux sollicitations d'alternance de température. La soupape fermée est déjà soumise à une différence de pression et de température entre l'admission et l'échappement.

En cas de débit, la différence de température peut s'accroître à $250^{\circ} \mathrm{K}$ par l'effet Joule-Thomson et par l'eau d'injection de refroidissement de la vapeur. Des chocs de température apparaissent lors de chaque ouverture de la soupape. Un choc thermique local dû̀ à l'eau non évaporée doit également être escompté en raison de l'injection intégrée. Les gradients de température de la vapeur vive au démarrage de la chaudière doivent aussi être observés.

Ces sollicitations complexes imposent au constructeur de configurer le corps de façon optimale :

- des corps de base sphériques pour les côtés haute et basse pression autorisent des épaisseurs de parois minimum et accroissent en conséquence la longévité cyclique;

- l'acier forgé $21 / 4 \mathrm{Cr} 1$ Mo possède le meilleur compor- 
tement aux chocs thermiques et réduit en conséquence la sensibilité aux criques;

- la forme la plus lisse possible, évitant les accumulations de matière, confère des pointes de tension minimales.

La cage brise-jet, la tige et le siège constituent d'autres composants critiques. Le genre de construction en $Z$ du corps permet de démonter et d'inspecter ces pièces sans démontage de l'entra înement.

La cage située sur le côté échappement a pour mission :

- d'assurer la protection du corps de la soupape contre les chocs thermiques dus à de l'eau non évaporée; - d'améliorer le mélange de l'eau d'injection et de la vapeur ;

- d'affiner les turbulences de sortie.

La cage est donc soumise à un double effort. Elle est excitée à la vibration par les coups de pression et les turbulences apparaissant lors de l'expansion et subit un choc thermique dû à l'eau non évaporée. La figure 4 montre de quelle façon une prolongation significative de la longévité a pu être obtenue en augmentant la rigidité de la forme.

Le siège interchangeable est également sollicité par le choc thermique et les différences de température sur son épaisseur. Une attention particulière a été ici observée lors du choix du blindage du siège en choisissant une matière possédant un grand pouvoir de déformation.

$\mathrm{Au}$ cours de ces dernières années, nos clients nous ont imposé des exigences croissantes de valeurs garanties de développement de bruit de plus en plus faibles. Les causes du bruit sont toujours dues à des oscillations de pression de la vapeur. Suivant la configuration du canal d'écoulement, la vitesse du son peut déjà être obtenue dans des conditions de pression largement sous-critiques lors de l'étranglement de la vapeur dans une soupape à un étage. Le jet généralement de forme annulaire, s'échappant du siège continue à se propager à une vitesse ultrasonique et passe à une vitesse subsonique avec formation de coups de compression. En outre, le jet est entouré de fortes turbulences qui se déplacent en aval. D'autres perturbations telles que des tourbillons de zones mortes et de décollement, apparaissent également. Les oscillations de pression résultantes se transmettent sur l'enceinte fixe et sont décelables sous forme de bruit et de vibrations mécaniques. Ces perturbations s'éten-
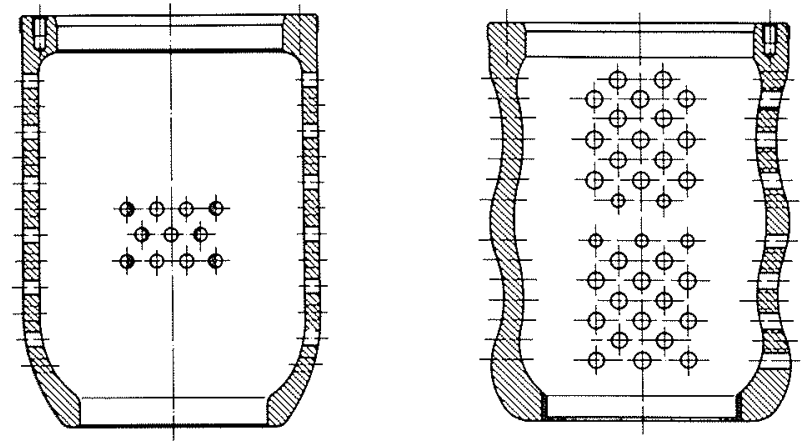

Figure 4. - Cage brise jet. dant sur une large gamme de fréquences, la probabilité que des parties fixes ou des espaces de vapeur soient excités en vibrations propres importantes est rela. tivement élevée.

Une réduction considérable des noyaux d'expansion - émetteurs de coups et d'ondes de pression - et un affinement de la turbulence sont obtenus du fait de la répartition du jet en expansion en plusieurs jets partiels.

Les effets de cette modification sur le spectre sonore d'une station de by-pass haute pression pour un débit effectif de $600 \mathrm{t} / \mathrm{h}$ sont représentés sur la figure 5 . Les pointes pénibles marquées ont disparu du fait de la répartition du canal annulaire en 16 canaux partiels (Fig. 6).

Les mesures de réduction du bruit se limitent non seulement à la construction de la soupape mais elles doivent constituer un programme pour l'ensemble de l'installation. En font par exemple partie: les tronçons de stabilisation de l'écoulement en amont et en aval de la soupape, les épaisseurs de parois surdimensionnées de la conduite côté échappement, l'élimination de coudes gênants, de raccords en $T$ ou de réductions à proximité immédiate de la soupape. En-dehors de ces mesures des plus simples, l'étude acoustique de l'ensemble du système est plus complexe et plus coûteuse.

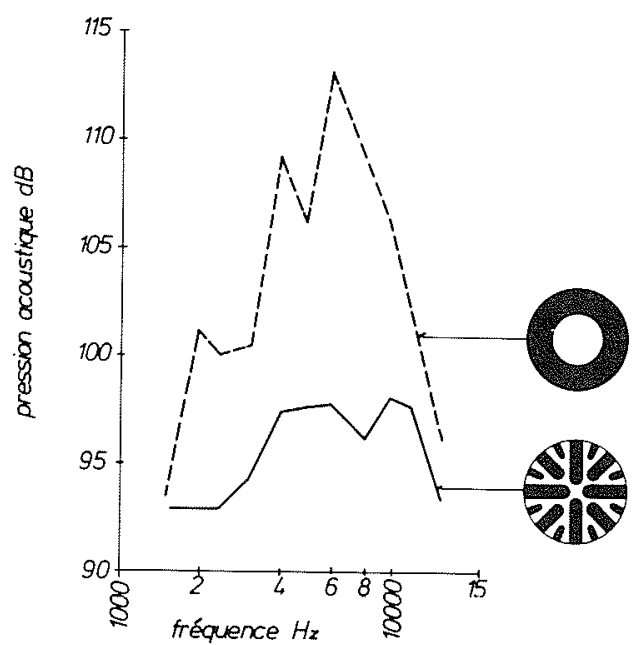

Figure 5. - Spectre acoustique pour soupape de détente avec tige à champignon et à fente.

\section{Mode de fonctionnement du système de com- mande et de régulation}

Le système de commande et de régulation est représenté sur la figure 7 . La soupape peut ainsi être soit positionnée avec la régulation analogique, soit ouverte rapidement avec le circuit de sûreté. L'ouverture complète de la soupape est atteinte en moins de 3 secondes avec l'ouverture rapide. Les valeurs effectives sont considérablement plus petites, comme nous le constaterons ultérieurement sur un exemple.

La figure 8 est une représentation schématique de la régulation analogique. La pression de la conduite de vapeur $H P$ est transmise par deux convertisseurs de pression indépendants l'un de l'autre (1). Les deux signaux de sortie sont comparés entre eux. Les écarts 


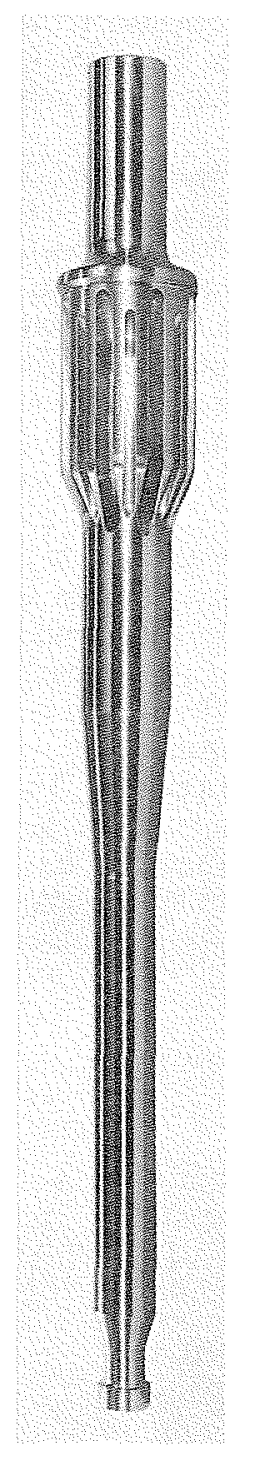

Figure 6

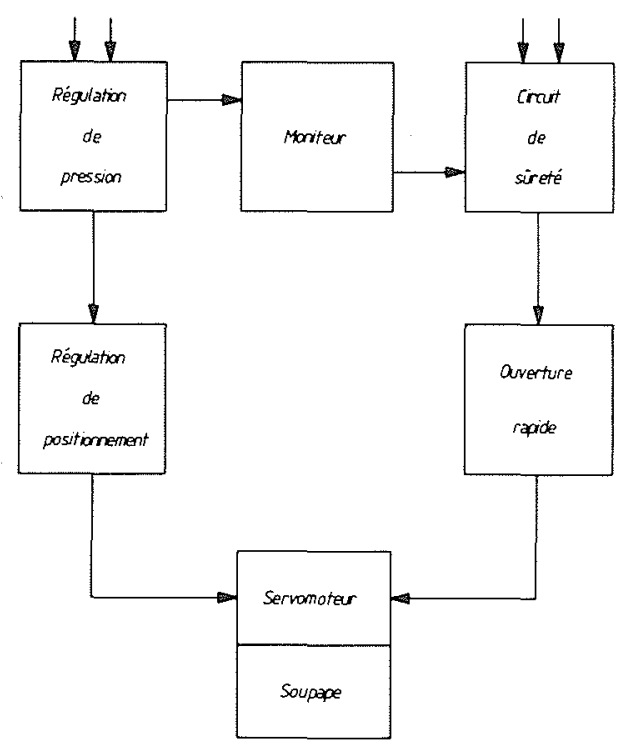

Figure 7. - Dispositif de régulation et de sûreté pour soupape de contournement haute pression

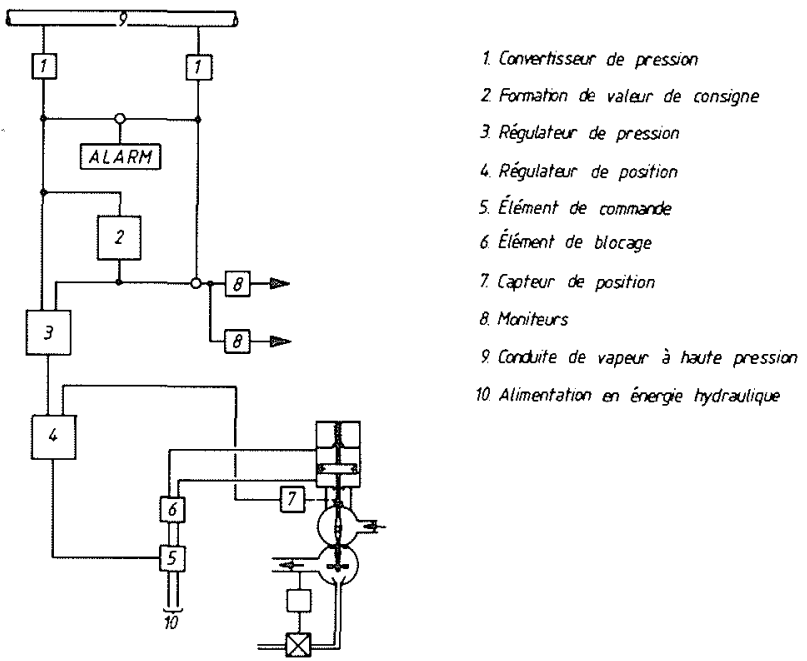

Figure 8. - Régulation analogique

qui dépassent une tolérance déterminée déclenchent une alarme. Un des deux signaux est alors utilisé pour la formation d'une valeur de consigne (2). En outre, un seuil constant est ajouté au signal d'entrée (donc à la pression mesurée), le gradient et la valeur maximale du signal d'entrée étant limités. La grandeur des seuils, ainsi que la grandeur du gradient et de la valeur maximale, sont ajustables en fonction des exigences de l'intallation. La valeur de mesure de la pression et la valeur de consigne sont alors amenées au régulateur de pression (3).

Cette commande fait que les soupapes de contournement sont fermées pendant le fonctionnement normal. Des variations de pression inférieures, situées a l'intérieur des limites ajustées ne provoquent pas d'ouverture de la soupape de contournement.

La sortie du régulateur de pression est amenée sous forme d'une valeur de consigne aux régulateurs de position (4) des différentes soupapes de contoumement et est comparée à la position de la soupape. En cas d'écart, l'élément de commande (5) serait manœuvré de façon correspondante. L'élément de blocage (6) est nécessaire pour fermer les conduites d'huile en cas de défaillance de l'alimentation en courant ou en huile. Il n'a pas d'influence sur la fonction de régulation normale. La position effective de la soupape est transmise à partir du capteur de position (7).

Ce système permet, dans le cas du mode de fonctionnement de contournement normal, de positionner les soupapes avec la régulation de pression.

Lorsque l'installation est utilisée avec une pression glissante, des mesures supplémentaires doivent être prises pour éviter un chargement de la chaudière dans le cas de délestages à basse pression. Cette mission est automatiquement assurée par la régulation de la pression suscitée par la formation de la valeur de consigne. Le système des moniteurs est prévu pour assurer une sécurité supplémentaire. Si la pression mesurée dans la conduite haute pression dépasse le signal de sortie de la formation de la valeur de consigne d'un montant prédéterminé, les deux moniteurs (8) du système de sécurité d'ouverture rapide interviennent. Une double redondance est obtenue par le fait que les deux moniteurs 
travaillent avec une valeur limite supérieure et une autre inférieure lors du dépassement desquelles, toutes les soupapes de contournement sont ouvertes. Le système de contrôle fonctionne selon le principe du courant de travail.

Le système de sécurité d'ouverture rapide est représenté de façon simplifiée sur la Figure 9. Contrairement au système des moniteurs, il fonctionne selon le principe du courant de repos, c'est-à-dire qu'une interruption du signal conduit à une ouverture des soupapes. Il est conçu avec trois circuits de signaux indépendants et travaille normalement suivant le principe "1 de 3". Une interruption d'un seul circuit provoque l'ouverture de toutes les soupapes.

Un système supplémentaire permet la vérification pendant le service.

La pression dans la conduite de vapeur HP est surveillée par 3 manostats de sûreté. Chaque manostat commande un des 3 relais de sécurité. Chaque relais de sécurité déclenche pour chaque soupape un des trois circuits hydrauliques d'ouverture rapide.

Les circuits hydrauliques d'ouverture rapide sont conçus de manière à ce que l'alimentation en huile ne soit pas nécessaire pour l'ouverture rapide. La force agissant sur la tige de la soupape (différence de pression sur le siège et le ressort) transfert le volume d'huile au servomoteur par les trois circuits hydrauliques d'ouverture rapide qui sont ouverts (Fig. 10).

L'action combinée de la régulation analogique et du système de sécurité d'ouverture rapide est très simple: lorsque la pression dans la conduite de vapeur $H P$ dépasse la valeur de consigne, les soupapes de contournement sont ouvertes de façon correspondante par la régulation analogique. Une poursuite de l'augmentation de la pression au-delà des valeurs limites des moniteurs déclenche l'ouverture rapide des soupapes.

Si l'augmentation de la pression chute à nouveau endessous des limites des moniteurs, la régulation analogique réassure le contrôle de la position des soupapes. Ce processus est indépendant de la position correspondante de la soupape.

Je souhaiterais prendre comme exemple de mes explications une chaudière à circulation forcée alimentée en lignite, à parois de la chambre de combustion hélicoi-

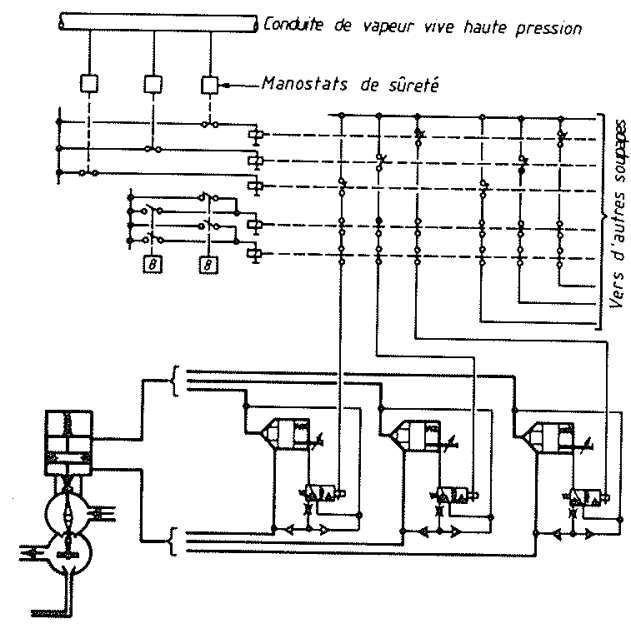

Figure 9. - Système de sureté et d'ouverture rapide

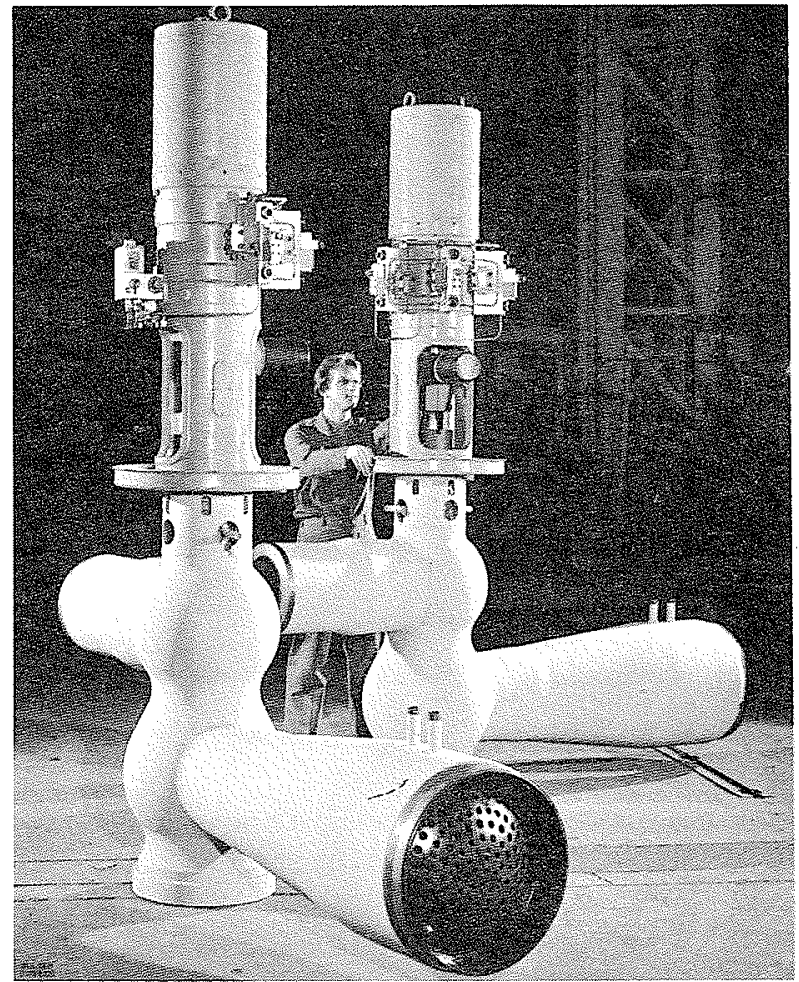

Figure 10

dales. La chaudière est conçue pour produire environ $1000 \mathrm{t} / \mathrm{h}$ de vapeur.

Dans ce cas, le système de contoumement $H P$ est constitué par trois soupapes de contoumement $H P$ du type ARS 112 à servomoteurs hydrauliques du type ASM $250 \mathrm{f}$. Une soupape de régulation d'injection du type A 20 à servomoteur hydraulique du type ASM 63 est affectée pour la régulation de la température à chaque soupape de contournement.

L'essai consistait en un délestage de la charge maximale (315 MW) au fonctionnement en ilotage (Fig. 11). La valeur de consigne de pression de la formation de la valeur de consigne avait été ajustée à 10 bars au-dessus de la valeur de pression mesurée. Le gradient de pression a été limité à 10 bars/minute. La valeur limite inférieure des moniteurs a été utilisée pour le déclenchement de la troisième soupape de contoumement et a été ajustée à 6 bars au-dessus de la valeur de consigne de pression, la valeur limite supérieure pour le déclenchement de la première et de la seconde soupapes de contournement à 8 bars au-dessus de la valeur de consigne de pression. Les manostats de sûreté ont été réglés sur 191, 192 et 193 bars.

La pression de service a été maintenue à 162 bars avant l'essai. Il en est résulté une valeur de consigne de pression de 172 bars et des valeurs limites des moniteurs de 178 et 180 bars.

Les valeurs de la pression enregistrées dans la conduite de vapeur haute pression et la position des trois soupapes de contournement sont représentées sur la figure 11 .

Environ 0,3 seconde après l'arrêt, la pression commence à s'accroître rapidement. Le gradient de pression 


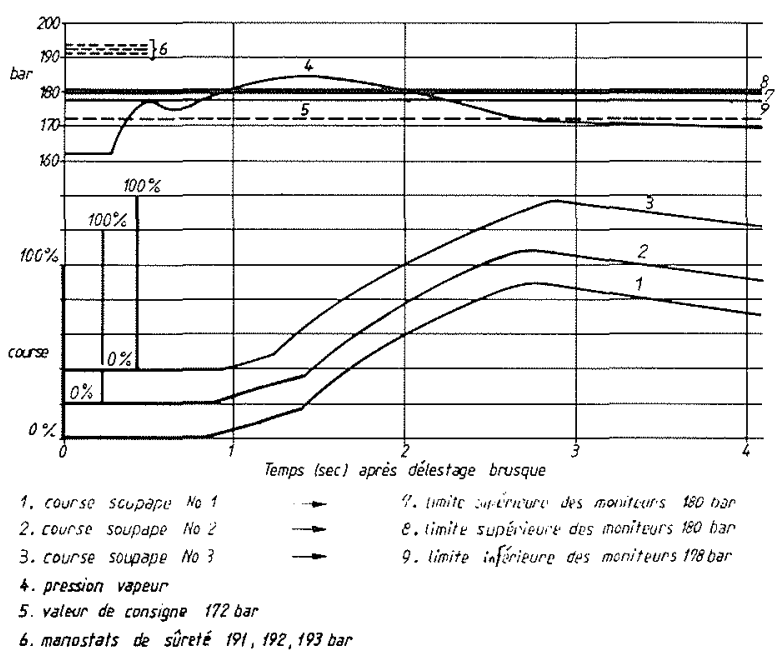

Figure 11. - Test de délestage brusque

maximal s'élève à 120 bars/seconde. Cette première onde de pression atteint une valeur maximale de 177 bars après environ 0,5 seconde. Après une chute à 175 , la pression commence à nouveau à s'accroître à une vitesse de 20 bars/seconde.

Les 3 soupapes de contournement ont commencé à s'ouvrir sous l'effet de la régulation analogique après environ 0,8 seconde. La vitesse d'ouverture s'élève à $30 \% /$ seconde. Cela donne 3,3 secondes pour la course totale de la soupape. Le système de sécurité d'ouverture rapide de la troisième soupape de contournement est déclenché après environ 1,2 seconde par la valeur limite inférieure des moniteurs. 0,2 seconde après, le système d'ouverture rapide de la première et de la seconde soupapes répond également. La vitesse d'ouverture s'élève avec l'ouverture rapide à 1,8 seconde pour la course complète de la soupape.

Sous l'effet de l'ouverture des soupapes de contournement l'augmentation de pression se ralentit et la pression atteint une valeur maximale de 184 bars après environ 1,3 seconde. Ensuite, la pression redescend à environ 172 bars. Pendant ce temps, la troisième soupape de contoumement atteint $98 \%$, la première et la seconde environ $85 \%$ de leur course totale.

I.e contrôle de la première et de la deuxième soupapes de contournement est ramené à la régulation analogique 2,6 secondes après le délestage. La même chose apparaît pour la troisième soupape 0,2 seconde après.
Ceci provoque une fermeture des soupapes à une vitesse de $13 \% /$ seconde (ce qui correspond à 7,7 secondes pour la course totale de la soupape). Les conditions de pression stationnaire sont obtenues après environ $15 \mathrm{se}$ condes. Dans ce cas, les trois soupapes de contournement sont ouvertes à environ $60 \%$.

Les programmes de calcul Rapid Flow et Reheat (RH)-Flow ont été développés pour calculer le comportement dynamique de la pression à la sortie de la chaudière et pour obtenir des indications pour l'ajustage nécessaire à cet effet du dispositif de sécurité des stations de contoumement $H P$ resp. des soupapes de sûreté. L'utilisation de ces programmes offre des avantages considérables par rapport aux essais des installations: - économie de coût ;

- éliminatiom du risque des essais sur des installations.

$\mathrm{La}$ principale mission des programmes se situe au niveau du calcul de l'écoulement de pression et de courant de vapeur dans les systèmes de resurchauffeur et haute pression après une modification rapide $\mathrm{du}$ courant de vapeur à la turbine, comme par exemple en cas de fermeture rapide de la turbine ou de fonctionnement en ilôtage.

Le système simulé dans le programme Rapid Flow comprend :

- un générateur de vapeur ;

- une ou plusieurs conduites de vapeur du générateur de vapeur à la turbine ;

- une ou plusieurs soupapes de contournement ;

- une ou plusieurs soupapes de sûreté.

Pour le calcul, les conduites de vapeur et le générateur de vapeur sont subdivisés en plusieurs tronçons. Le main tien de la masse et de l'impulsion est appliqué dans chaque tronçon. Le programme RH-Flow comprend:

- un ou plusieurs resurchauffeurs et les conduites correspondantes;

- une station de contournement basse pression;

- une soupape de sûreté de resurchauffeur.

Le volume de vapeur de l'ensemble du domaine moyenne pression est regroupé dans un tampon fictif pour le calcul.

Le comportement de la pression en fonction du temps dépend de la différence entre l'ensemble de l'admission et de l'échappement

Les soupapes de contoumement et de sûreté sont simulées dans les deux programmes y compris leur commande et la régulation, avec retards correspondants. 


\section{Discussion}

Président : M. R. CARDINAL

Le Président. - Je remercie M. GROTLOH de sa communication.

Je pense qu'elle va susciter des questions, soit sur le principe mêrne de cette soupape combinée, soit sur sa technologie, soit sur le principe de sa commande.

M. LEPHILIBER T. - Comment forgez-vous un corps de cette forme?

M. GROTLOH. - Il y a plusieurs possibilités. Normalement, on forge une pièce ronde qui, après, est usinée selon les contours extérieur et intérieur. Pour éviter des épaisseurs trop grandes à l'origine, on peut même, pour commencer, pour les très grandes pièces, forger cela sur un poinçon. A l'intérieur, il y a déjà un trou. Les entrées et les sorties sont ensuite soudées.

Le Président. - Est-ce que le fait que la garniture soit constamment sous pression ne pose pas un problème?

M. GROTLOH. - Non. Ces vannes sont construites pour des pressions de 215 bars et des températures de $545^{\circ}$ Celsius. Elles sont construites pour toute la durée d'une centrale.

M. CHEVALIER. - Il est bien connu que ces vannes de contournement de turbines sont parmi les plus bruyants des matériels des centrales.

Quelles dispositions avez-vous prises à la conception pour prévoir le niveau de bruit à l'émission de telles vannes?

Quelles dispositions prenez-vous dans leur installation?

A quelles garanties êtes-vous tenu en bruit global ?

M. GROTLOH. - Comme je l'ai montré, on a pu réduire le bruit à 97 décibels. Mais, naturellement, il peut y avoir des influences de la tuyauterie. Pour ces cas-là, nous avons des spécialistes à mêtme de prévoir les dispositions à adopter pour réduire ces influences.

M. CHEVALIER. - Le spectre que vous montrez est-il un spectre normalisé à $1 \mathrm{~m}$

M. GROTLOH. - A $1 \mathrm{~m}$ de distance, en décibel A.

Le Président. - Je suppose que les soupapes de sécurités en aval du resurchauffeur sont capables du plein débit ?

M. GROTLOH. - Oui.

Le Président. - Vous prenez également en considération le fait que l'injection d'eau ne peut pas non plus être toujours assurée en conditions incidentielles.

M. GROTLOH. - C'est exact. 